\title{
Visceral Leishmaniasis: A Hidden Killer?
}

Jayasena $W_{M A S^{1}}$, Abeygunasekara $\mathrm{PH}^{2}$, Jayasundara $\mathrm{JP}^{1}$,

${ }^{1}$ Office of the Judicial Medical Officer, District General Hospital, Matale

${ }^{2}$ Department of Pathology, Teaching Hospital, Anuradhapura

${ }^{*}$ Corresponding author: Tel: 0094-773438345. E-mail address:

ajithjayasena@yahoo.co.uk

MLJSL. Vol 1. No 2. Aug. pp $37-41$

\section{Abstract}

Leishmaniases are parasitic diseases caused by protozoan flagellates of the genus Leishmania, parasites infecting numerous mammal species, including humans. This is transmitted through the infective bite of an insect vector, the phlebotomine sandfly.

The leishmaniases are threatening 350 million people in 88 countries of four continents. In Sri Lanka too, cutaneous manifestation of leishmaniasis has been reported particularly in North Central Province.

Visceral leishmaniasis is a disease of the mononuclear phagocytic system, commonly affecting the spleen, liver, lymph nodes and bone marrow. However, other organs (intestine, lung) and skin may also become involved. In advanced cases, as in immune suppressed patients all organs are involved.

This case revealed a death of a 47 year old deceased male who was presented to the hospital with an upper abdominal pain for eight days and ultra sound examination revealed splenomegaly. Additional information indicated he had had on and off abdominal pain for one year, and the investigations revealed low haemoglobin, neutropenia with no features of malignancy and screening for haemoglobinopathy was suggested.

At the autopsy massive splenomegaly, hepatomegaly and ascites were observed and sections from all organs were taken for histology, which revealed Leishmania donovani bodies in Kupffer cells with dense perivascular infiltrate of lymphocytes and plasma cells. Spleen too, showed the sinuses that contain macrophages with LD bodies.

Conclusion: This case demonstrates visceral leishmaniasis as the cause of death and this is the first autopsy case where such a cause of death was encountered in Sri Lanka and reinforces the need of awareness of leishmaniasis.

Key words: Visceral leishmaniasis, Leishmania donovani, LD bodies. 


\section{Introduction}

Leishmaniases are parasitic diseases caused by protozoan flagellates of the genus Leishmania, parasites infecting numerous mammal species, including humans. This is transmitted through the infective bite of an insect vector, the phlebotomine sandfly [1].

The leishmaniases are threatening 350 million people in 88 countries of four continents [1] . In Sri Lanka too, cutaneous manifestation of leishmaniasis has been reported in five provinces (central, north central, north western, northern and eastern), with the possibility of it being a zoonosis [3] Though VL is endemic in our neighbouring countries such as India and Bangladesh for many decades the first case of VL was reported in Sri Lanka in 2007 [2] It has three main clinical forms, visceral, muco-cutaneous and cutaneous. Most leishmaniases are zoonoses and the reservoir hosts are various species of mammals, either a domestic or wild mammals [1]. Therefore, more studies are needed for evidence of reservoir(s) and identification of behavior of the vector species in order to explain the atypical presentation of L.donovani in Sri Lanka[3].

This case revealed a death of a 47 year old male who presented to the hospital with an upper abdominal pain for eight days with past history of on and off abdominal pain for one year. The diagnosis of visceral leishmaniasis was established by histology following the autopsy examination.

\section{Circumstances and autopsy findings}

A 47 year old male was admitted to the hospital with a history of generalized abdominal pain with distension for eight days. He was a chronic alcoholic and an unskilled labourer. There was no history of overseas stay and he was afebrile and pale on admission. His haemoglobin was $4.6 \mathrm{~g} / \mathrm{dl}$ and blood picture revealed hypochromic, microcytic red cells with marked rouleaux formation white cells showed mild leucopenia.

There was no evidence of haematological malignancies, and. Screening for haemoglobinopathy was suggested. Other investigations performed were full blood count $10.4 \times 10^{3}$, blood urea $70 \mathrm{ml} / \mathrm{dl}$, SGPT 57 iu (4-47) and SGOT 174 iu (normal 4-42). Ultrasound scanning of the abdomen revealed an enlarged spleen with ascites and no lymphadenopathy. Liver biopsy was taken and on the following day he died.

Autopsy revealed a moderately nourished middle aged male with distended abdomen. He was pale and there were no skin lesions, such as ulcers, pigmentation, patchy areas of bleeding or muco-cutaneous lesions. Examination of abdomen revealed a biopsy site on the surface of the liver and no haemoperitoneum, but approximately 500 $\mathrm{ml}$ of straw coloured ascites was detected. Spleen was enlarged, firm and weighed $2510 \mathrm{~g}$ and the liver was enlarged, firm with no focal lesions weighing $1525 \mathrm{~g}$. There was no lymphadenopathy.

Histological sections with H\&E stain revealed the liver was densely inflammed with perivascular infiltrate of lymphocytes and plasma cells. The Kupffer cells within the sinuses contained numerous Leishmania donovanni bodies (LD bodies). Sections of the spleen showed congestion and sinuses contained numerous macrophages with LD bodies. Sections of kidneys, heart, lungs and brain were unremarkable. Histological features were consistent with visceral leishmaniasis. 


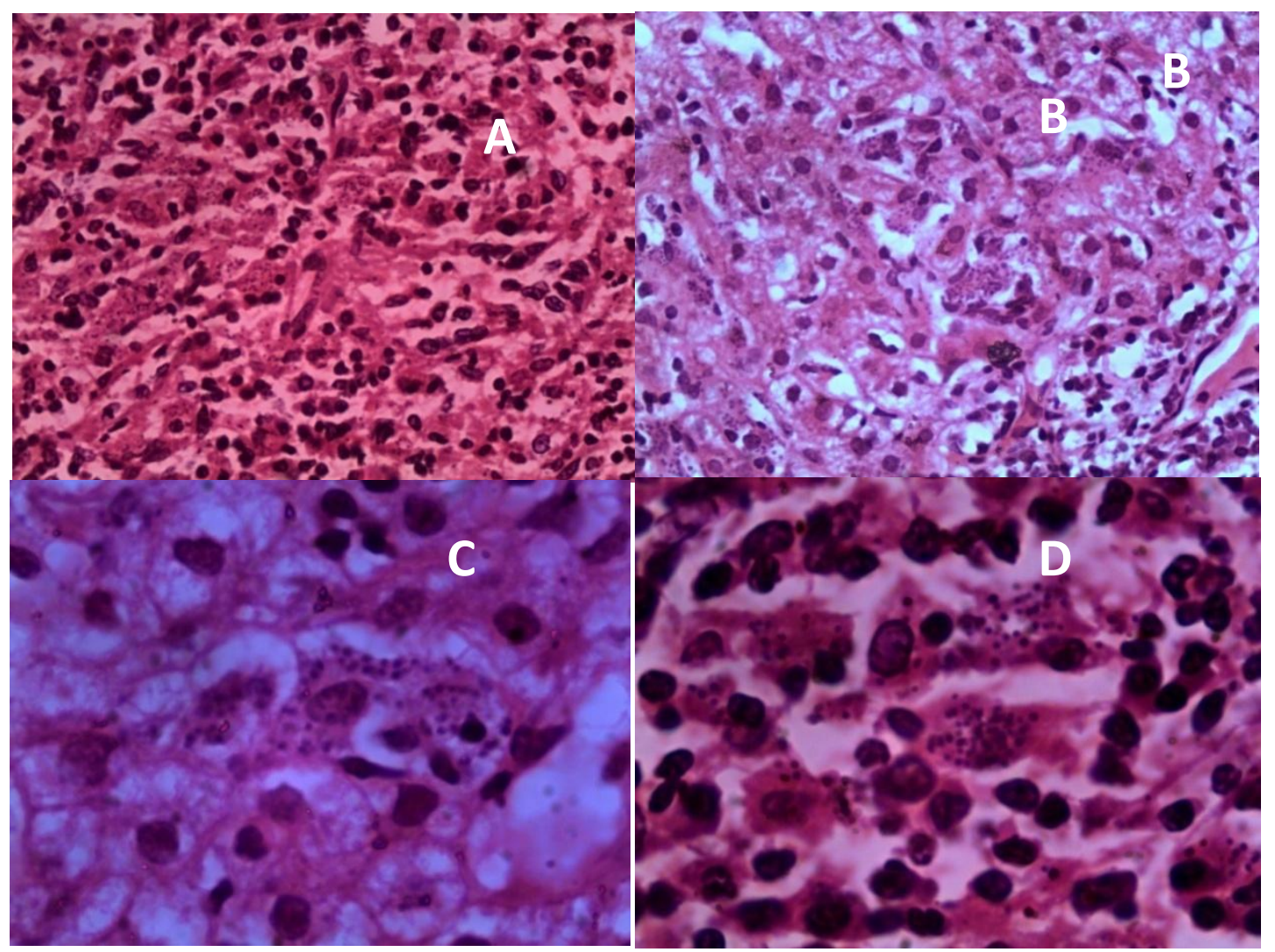
A. Liver $x 400 \mathrm{H} \& \mathrm{E}$ : LD bodies within Kupffer cells
B. Liver $x 1000$ H\&E: LD bodies within Kupffer cells
C. Spleen $\times 400 \mathrm{H} \& \mathrm{E}$ : LD bodies within macrophages
D. Spleen $\times 1000 \mathrm{H} \& \mathrm{E}$ : LD bodies in macrophages

\section{Discussion}

Visceral leishmaniasis is a disease of the mononuclear phagocytic system, commonly affecting the spleen, liver, lymph nodes and bone marrow. However, other organs (intestine, lung) and skin may also become involved. In advanced cases, as in immune suppressed patients all organs are involved [1]. However, in this case only spleen and liver were involved. The localization of the parasite to the various organs of the patient results in the clinical expression of the disease. In that sense, L.donovani and
L.infantum are viscerotropic and other species are dermotropic [1].

VL evolves slowly over many months or even a few years. A general syndrome including fever, asthenia, weight loss, anaemia, splenomegaly, hepatomegaly and sometimes adenopathia dominates the classical presentation [1]. Fever is the major symptom in acute presentations as well as in the chronic insidious forms[1], but it was not present with the deceased. The main clinical presentation of the deceased was on and off abdominal pain with gradual 
distension for approximately one year and he had not sought medical advice until the late stage. However, asymptomatic and subclinical forms are frequent and it makes a therapeutic dilemma for the clinician[1].

In VL splenomegaly appears early and is almost invariably present. Spleen size increases regularly, in relation to the duration of the disease. Eventually the spleen may extend down into the left hypochondrium[1] as detected in this case.

Hepatomegaly is less frequent in VL and occurs later than splenomegaly. The liver is generally slightly enlarged[1], but it was moderately enlarged in this case weighing 1525 g. Rarely jaundice appears in late stages which is considered of poor prognosis[1]. Jaundice was not seen in this case and there was no fatty change though he was said to be a chronic alcoholic.

Ascites is considered as a late sign of bad prognosis, sometimes associated with oedema and pleural effusion. These unusual signs are more common in Indian leishmaniasis[1].

Diarrhoea is frequently reported and is related to the ulcerations of the digestive mucosa which was not seen in this case. In India, patient's skin has a greyish pigmentation, which gives rise to the local name of the disease (kala-azar= black fever) [1]. In this case, anaemia is responsible for paleness of the mucosa.

Furthermore, the deceased did not show cutaneous manifestations such as skin ulcers, which have been detected with increasing frequency in Sri Lanka[3,4], where L.donovani has been identified as the causative agent [5].
When the phagocytic cells are overloaded with parasites it predisposes the patients to bacterial infections which is the usual cause of death [6]. Haemorrhages related to thrombocytopaenia may also be fatal, [6] but it was not evident here. In the absence of other natural causes, haemorrhage and sepsis in this case, the cause of death can be concluded as Visceral Leishmaniasis and low haemoglobin and asites indicates that the deceased suffered cardiac failure which led to death. Finally this case demonstrates an unusual cause of death in medico-legal investigation of deaths in Sri Lanka and reinforces the need of awareness of visceral leishmaniasis.

\section{Acknowledgement}

The authors wish to extend their sincere thanks to Dr.Kumari Dissanayake for retrieving medical records of the deceased and for the secretarial work.

\section{References}

1. Cook GC, Zumla A. Manson's Tropical Diseases. London: WB Saunders; 2003. P. 1339-1364.

2. Abeygunasekara PH, Costa YJ, Seneviratne N, Ratnatunga, N, Wijesundera M de S. Locally acquired visceral leishmaniasis in Sri Lanka. The Ceylon Medical Journal 2007; 52: 30-1.

3. Navarathna SSK, Weilgama DJ, Rajapksha K. Cutaneous leishmaniasis in Sri Lanka: a study of possible animal reservoirs. International Journal of Infectious diseases 2009;13: 513517.

4. Cutaneous leishmaniasis, Sri Lanka. Emerging Infectious Diseases. www.cdc.gov/eid. vol 13,(07). July 2007

5. Karunaweera ND, Pratlong F, Siriwardana HV, Ihalamulla RL, Dedet JP. Sri lankan cutaneous 
leishmaniasis is caused by Leishmania donovani zymodeme MON-37. Trans $R$ Soc Trop Med Hyg. 2003;97:1-2.

6. Kumar V, Abbas AK, Fausto N. Robbins and Cotran Pathologic Basis of Disease. Elsevier Saunders; 2005. Pp 403-404.

\section{Contribution of authors}

Performing the autopsy- WMASJ, JPJ

Opinion- WMASJ, PHA,

Writing the manuscript - WMASJ

Revising the manuscript- WMASJ 